\title{
Instituciones: Una mirada general a su historia conceptual ${ }^{*}$
}

\author{
Nivia Marina Brismat ${ }^{1}$ \\ Universidad Autónoma de la Ciudad de México (México)
}

Recibido: agosto 30 de 2014 - Revisado: noviembre 19 de 2014 - Aceptado: diciembre 17 de 2014

Referencia formato APA: Brismat, N. M. (2014). Instituciones: Una mirada general a su historia conceptual. Revista Cientifica Guillermo de Ockham, 12(2), 31-40.

\section{Resumen}

Este artículo tiene como objetivo principal reconstruir la evolución del concepto “institución” y de los estudios institucionales en el contexto de las ciencias sociales. Como es ampliamente conocido, en las últimas décadas ha tenido lugar una verdadera explosión de los debates e investigaciones sobre las instituciones, su origen, evolución e influencia en la sociedad. Estos esfuerzos han actualizado el debate sobre los logros y limitaciones de los enfoques institucionalistas, y el desafío de postular una teoría de las instituciones de carácter interdisciplinario que articule las diferentes propuestas teóricas sobre el tema. En ese contexto, este trabajo reconstruye la evolución del concepto "instituciones": su origen histórico, principales desarrollos, los enfoques institucionalistas contemporáneos, y sus principales logros y nudos problemáticos. A pesar de la heterogeneidad y limitaciones de los enfoques institucionales se vislumbran posibilidades para la construcción de un campo teórico "institucionalista" compartido, con una influencia innegable en el análisis social.

Palabras clave: Instituciones, análisis institucional, neoinstitucionalismo, teoría de las instituciones, ciencias sociales

\section{Institutions: An overview to its conceptual history}

\section{Abstract}

In this article, the author stresses the evolution of the concept "institution" and institutional studies in social sciences. In recent decades, there has been an explosion of institutions analysis and research: their origin, evolution and social influence. These efforts have updated the debate about the achievements and limitations of institutional approaches, and the challenge to advance an institutions interdisciplinary theory. This paper hopes to contribute to this debate from the reconstruction of the evolution of the term "institutions": its historical origins and main developments to date, the emergence of contemporary approaches, and the main achievements and problems of institutional approaches. The study highlights the fact that, although the heterogeneity and limitations of the institutional approaches, it is possible to construct a shared theoretical field about institutions in social analysis.

Keywords: Institutions, institutional theory, institutional analysis, new institutionalism, social sciences

* Este artículo se deriva de la participación en la investigación y escritura del Diccionario de justicia, como parte del proyecto Los límites de la confianza: justicia, derecho y cultura cívica (código 134051). Financiado por el Consejo Nacional de Ciencia y Tecnología (Conacyt). Responsable: doctor Carlos Pereda. Coordinadores: María Teresa Muñoz, Sergio Ortiz Leroux y Julieta Marcone (México) y varias universidades. Fecha de inicio: 16 de junio de 2011. Fecha de culminación: 15 de noviembre de 2014.

1. Doctora en Ciencias Sociales con especialización en Ciencia Política en la Facultad Latinoamericana de Ciencias Sociales (Flacso), sede México. Actualmente, es profesora-investigadora en la Universidad Autónoma de la Ciudad de México (UACM), Academia de Ciencia Política y Administración Urbana. Pertenece al grupo de investigación "Justicia, derechos y política social" en la línea de investigación "Pobreza, derechos y programas sociales”. E-mail: nmbrismat@yahoo.com nivia.marina.brismat@uacm.edu.mx 


\section{Introducción}

En los últimos veinte años hemos asistido al auge del concepto "institución" en todas-sino en la mayoría-de las disciplinas sociales. Este "redescubrimiento" nos ha llegado a través de una infinidad de enfoques y posiciones teóricas que han hecho del concepto un referente imprescindible, a la par que una suma heterogénea de elementos.

Actualmente, a pesar de que los enfoques institucionalistas han logrado posicionarse como una perspectiva relevante, se observan limitaciones para adelantar una teoría de las instituciones (consistente, sistemática, universal) en las diferentes disciplinas sociales y lo que quizá es más importante, construir un campo teórico común sobre el tema a partir de la mixtura interdisciplinaria.

Todo lo anterior permite adelantar una serie de interrogantes: ¿cuál es el origen del concepto "institución”?, ¿cuáles han sido sus principales contenidos y usos dentro de las ciencias sociales?, ¿̨cuáles son sus principales desarrollos teóricos en la actualidad? Y finalmente, ¿̨cuáles son los principales logros y nudos problemáticos en el tratamiento de este concepto?

Este artículo pretende adelantar algunas respuestas a las preguntas planteadas, con lo cual espera enriquecer las reflexiones y debates sobre un área de conocimiento en plena efervescencia. Asimismo, reconstruye elementos relevantes de la "historia conceptual" de un término extraordinariamente complejo, pero imprescindible para comprender muchos de los fenómenos sociales que le otorgan sentido a eso que denominamos "sociedad".

El trabajo se ha organizado de la siguiente manera: en primer lugar, se reconstruye la evolución y contenidos principales del concepto institución, con especial énfasis en aquellas disciplinas o ramas del saber social que han desarrollado de manera más sostenida el concepto. Seguidamente se describen y analizan los enfoques contemporáneos sobre las instituciones y, a modo de conclusión, se subrayan algunas de sus potencialidades y limitaciones.

\section{Trayectoria del concepto "institución" en las ciencias sociales}

El término institución proviene de la raíz indoeuropea esta, que significa "estar", "permanecer", pero también denota "poner", "colocar". De acuerdo con Herrera-Gómez y Castillo (2004), el significado del término alude a todo aquello que está objetivamente en el mundo (más allá de nuestras acciones y deseos) y a todo lo que se establece en el mundo debido a la voluntad humana.

La palabra tomó cuerpo en el latín a través de los verbos stere y sistere y los sustantivos statum, constitutio e institutio, que significan "poner", "fijar", "establecer", "edificar"; "regular", "organizar"; o "instruir", "enseñar", "educar" (Diccionario Jurídico Mexicano, 1988, p. 1745). Adicionalmente, los jurisconsultos romanos utilizaron el término institutiones o institutas para referirse a los libros o manuales elementales destinados a la enseńanza introductoria del derecho, como los escritos por Gayo en el siglo II (Gai Institutiones o Gaii Institutiones), y los de Teófilo y Doroteo (Institutiones Iustiniani o Institutiones Justiniani) (Vargas, 2001). Todo lo anterior, indica que la diversidad de usos y significados del término "institución", tanto a nivel cotidiano como en el campo del saber científico, derivan del carácter polisémico de su matriz etimológica.

El nacimiento y desarrollo de las diferentes ciencias y disciplinas del saber sobre la sociedad trajeron consigo múltiples tratamientos del concepto, no solo en las actualmente conocidas como ciencias sociales, sino también en la historia, la filosofía y las disciplinas jurídicas. A continuación, se reconstruyen sus tratamientos más relevantes en la sociología, la teoría de la organización, la economía y la ciencia política. ${ }^{2}$

El concepto de institución ha acompañado a la sociología desde sus orígenes hasta nuestros días. Emilio Durkheim, uno de los padres fundadores de la disciplina, selló el vínculo inextricable entre instituciones y sociología al lanzar una afirmación categórica sobre su objeto de estudio: la sociología es la "ciencia de las instituciones, de su génesis y funcionamiento" (Durkheim, 2001, p. 31).

Durkheim concibe a las instituciones como hechos sociales, esto es, como aspectos de la experiencia colectiva que se materializan en una multiplicidad de formas e instancias: el Estado; la familia; el derecho a la propiedad; el contrato; las tradiciones culturales, políticas y religiosas, etc. En suma, "se pueden llamar institución a todas las creencias y todos los modos de conducta instituidos por la comunidad" (Durkheim, 2001, p. 31).

Las instituciones no se ajustan completamente a cualquier situación o experiencia individual. En cuanto

2. Aunque la reflexión sobre las instituciones ha ocupado un lugar relevante dentro de muchas disciplinas sociales y humanísticas, nos hemos enfocado en aquellas que, en términos históricos, han hecho del análisis institucional un área de estudios relevante en sus campos respectivos. Se espera incorporar los aportes de otras disciplinas (la filosofía, el derecho, etc.), en investigaciones posteriores. 
prácticas o experiencias colectivas tienen la particularidad de que se constituyen y experimentan como "externas" a los individuos $y$, en ese sentido, tienen un papel constreñidor, incluso coercitivo, más allá de nuestras voluntades y deseos. Esta visión tendrá profunda influencia en los enfoques institucionalistas contemporáneos, al sostener que las instituciones se constituyen como barda y armazón de la acción humana: "Las instituciones se nos imponen, nosotros nos atenemos a ellas; nos obligan y nosotros las asumimos; nos constriñen y nosotros sacamos provecho de su funcionamiento y de la coacción misma que ejercen sobre nosotros" (Durkheim, 2001, p. 28).

Otro referente imprescindible en el abordaje del concepto "institución" lo constituye Max Weber. Aunque de forma más concisa, este autor define a las instituciones a través de un concepto susceptible de ser utilizado en todos los niveles sociales, esferas de acción o tiempos históricos: "las instituciones son relaciones sociales cristalizadas en el tiempo" (Weber, 2005, p. 12).

Weber concibe a las instituciones, en el lenguaje de su sociología comprensiva, como formas sociales específicas (Estado, cooperativas, leyes, sociedades anónimas, burocracias, etc.), en cuanto desarrollos y entrelazamientos de acciones de personas individuales. Son el desarrollo, en una forma determinada, de la acción social de unos cuantos individuos, bien sea real o construida como posible (Weber, 2005).

En la primera mitad del siglo XX, la influencia del funcional-estructuralismo marcó notablemente el pensamiento sociológico y, con ello, la forma como fueron pensadas las instituciones en la disciplina.

Talcott Parsons es la figura más relevante de esta escuela sociológica, que si bien no adelanta una teoría de las instituciones presenta algunas nociones y conceptos que se deben tener en cuenta para materializarla. Para este autor, en toda sociedad existe un sistema coherente de actitudes basadas en valores últimos comunes y las instituciones son una expresión de esas actitudes en ciertas relaciones particulares de la acción. Específicamente, tienen como función "la regulación de la acción de tal forma que mantenga la conformidad relativa con los valores comunes últimos de la comunidad" (Parsons, 1990, p. 331). En su interrelación, constituyen un sistema organizado de normas y creencias culturales comunes a la mayoría de los individuos que componen una sociedad (marco institucional) (Parsons, 1990; 1982).

A mediados del siglo XX la sociología se distanció del enfoque funcional-estructuralista para proveer un análisis de la interacción entre el contexto institucional y los agentes individuales, con el consiguiente desarrollo de estudios microsociológicos y el establecimiento de puentes con otras disciplinas sociales.

Ello tuvo gran influencia en la forma como se pensaron las instituciones, pues se enfatizó el papel del individuo y sus relaciones más inmediatas en la producción y reproducción institucional. Así, Homans postula la teoría del intercambio, que considera que los individuos y su comportamiento son relevantes para comprender la sociedad y de su interacción emergen las normas y regulaciones sociales basadas en la aprobación social (Homans, 1961).

La aparición de la sociología económica, como puente entre la sociología y la economía, adelantó una serie de elementos relevantes en el estudio de las instituciones a nivel microsocial. Su existencia como producto del consentimiento de actores racionales implica la revisión de dos conceptos: El concepto de social embeddedness, referido al grado en que la actividad económica es constreñida por instituciones no económicas (Granovetter, 1985). El concepto de capital social, en cuanto conjunto de recursos y posibilidades sociales que abren oportunidades a los individuos y las instituciones se constituyen como forma relevante para capturar las ganancias de la cooperación (o para destruirlas) (Coleman, 1988a, 1988b; Nee \& Ingram, 1998).

El desarrollo de la sociología económica se debió, en buena medida, a la influencia de la obra Veblen (para mayor detalle ver Stinchcombe, 1997). Esta perspectiva ha sido ampliamente recuperada y desarrollada en los enfoques contemporáneos sobre las instituciones (Merton, 1998).

En los primeros estudios institucionales basados en el análisis organizacional se destacan los trabajos de Selznick (1948; 1957). En sus trabajos sobre la autoridad del Valle de Tennessee subraya la importancia de los elementos informales en las organizaciones, como las normas, creencias y valores de los miembros, que comienzan a formar parte y a reproducirse (a institucionalizarse) como parte de la lógica de la organización, más allá de sus elementos formales (Selznick, 1949). Este autor arriba a una conclusión fundamental: la trayectoria y permanencia de una organización no responde solamente a cuestiones instrumentales de medios-fines, sino también a la institucionalización de una identidad compartida: "Institucionalizar es infundir valores más allá de los requerimientos técnicos de la tarea presente" (Selznick, 1957, pp. 16-17). 
Si trasladamos el análisis a la economía, el antecedente principal de los estudios institucionales en la disciplina corresponde a la escuela denominada "economía institucional". Con este término, propuesto por Walton Hamilton en 1919 en la American Economic Association (Rutherford, 2001, p. 173), se agruparon una serie de contribuciones que iniciaron a finales del siglo XIX y cuyo florecimiento tuvo lugar en el período entreguerras. Dentro de los autores agrupados en esta tendencia, sobresalieron Thorstein Veblen, John Commons, John Clark, y Wesley Mitchell (Gruchy, 1972; Hodgson, 1998; Rutherford, 2001).

Los trabajos e influencia de Veblen fueron determinantes para dotar a la economía institucional de algunos de sus rasgos más característicos. Para este autor, las instituciones son mecanismos que modelan a los individuos; es decir, son formas enraizadas que guían el comportamiento y el pensamiento y determinan las preferencias y valores individuales, así como las características del sistema económico (Hodgson, 1998).

En sus diversos trabajos sobre la economía estadounidense, Veblen criticó el interés por las ganancias y el éxito pecuniario como valores centrales del sistema económico. Asimismo, cuestionó la viabilidad de "la mano invisible del mercado", las pérdidas asociadas al monopolio, las estrategias empresariales manipuladoras, etc. En muchas ocasiones, estos cuestionamientos se combinaron con una posición más radical que postulaba suplantar el mercado por otras formas de "control social" que condujeran a un mayor bienestar social (Veblen, 1974).

Otra gran corriente de la economía institucional se centró en la relación entre Economía y derecho. Con base en los postulados de la escuela histórica alemana, sobresalieron los trabajos de Hamilton, Clarck, Hale y Commons. Este último analizó la relevancia de las legislaturas y las cortes para resolver los conflictos, y la evolución de la ley como resultado de esos procesos. A nivel micro, desarrolló el concepto de transacción como unidad básica de análisis (Commons, 1932; 1934), concepto de gran relevancia en los enfoques institucionalistas contemporáneos.

En su conjunto, la economía institucional hizo grandes contribuciones, dentro de las cuales destacan los estudios sobre el ciclo de los negocios, el comportamiento de los precios en las empresas, el monopolio y la competencia, los sindicatos y los mercados laborales, las fallas de mercado, las utilidades públicas y la regulación, además de avanzar en las mediciones econométricas y en los vínculos entre derecho y economía. La idea de que, en ciertas circunstancias, la competencia puede ser destructiva o acarrear consecuencias fatales, es común entre los institucionalis- tas, así como la idea de la inadecuación de los modelos estándares de la competencia perfecta (Rutherford, 2001).

A pesar de haber tenido una etapa de florecimiento-especialmente en el período entreguerras- esta escuela pierde prestigio y posición. Ello se relacionó, entre otros factores, con su incapacidad para ofrecer una visión alternativa sobre el comportamiento humano que permitiera abandonar los supuestos de la economía neoclásica ortodoxa. Con el renacimiento del interés por las instituciones en economía, se han aplicado los términos "vieja economía institucional" o "viejo institucionalismo" a esta perspectiva, a pesar de reconocerse su influencia y recuperar conceptos centrales como el de "transacción".

En el campo de los estudios políticos, por su parte, existe un amplio consenso en el sentido de que la preocupación por las instituciones políticas es tan antigua como las reflexiones filosóficas sobre esta esfera. Una larga lista de pensadores (desde Platón y Aristóteles) han hecho suya la preocupación por las instituciones políticas y sus efectos en la sociedad (Rothstein, 2001).

Con la configuración del campo de los estudios políticos a finales del siglo XIX e inicios del XX, el análisis de los diseños constitucionales y el interés por la forma institucional del gobierno (organigramas, departamentos, oficinas, etc.) concentró la atención de los politólogos, además de la preocupación por el "buen gobierno", es decir, por las normas y valores que deben orientar el quehacer gubernamental (Goodin, 2003; Peters, 2003).

La revolución conductista, con una notable influencia en el derrotero de la disciplina en pleno siglo XX, promovió el énfasis en el comportamiento individual y la búsqueda de construcciones más sofisticadas teórica y analíticamente, con lo que se relegó relativamente el estudio de las instituciones en ciencia política (Rothstein, 2001). Sin embargo, impulsó el desarrollo de la perspectiva de la elección racional en la disciplina que, a partir de los supuestos y lenguaje de la economía, hizo notables avances en los estudios sobre el comportamiento político.

A partir de los trabajos seminales de Arrow (1951), Downs (1957), Buchanan y Tullock (1962) y Olson (1965) se desarrolla una perspectiva interesada por las elecciones que transcurren fuera del mercado, a partir del supuesto de que, tal y como ocurre en la esfera económica, los individuos en el mundo de la política son maximizadores de la utilidad y se guían por el autointerés, ya sean votantes, políticos o burócratas (Caporaso \& Levine, 1992; Shepsle \& Bonchek, 2005). Cimentadas en esa matriz teórica, las instituciones se ajustan al cálculo y la 
elección de los individuos. En todo caso, poseen alguna relevancia e influencia sobre los participantes al establecer parámetros para el comportamiento individual coml reglas que permiten la eficiencia o el óptimo social $(\mathrm{Bu}-$ chanan \& Tullock, 1962). Este enfoque ha contribuido de manera relevante a la comprensión de las instituciones actualmente.

\section{Desarrollos contemporáneos: la emergencia de los "nuevos institucionalismos"}

Desde finales del siglo XX hasta la actualidad, ha resurgido el interés por las instituciones en diversas disciplinas y subdisciplinas sociales. Estas, con sus diferencias, incluyen a las instituciones como variables endógenas del análisis, aceptan que el tejido institucional influye en las prácticas de los individuos en diferentes niveles y, en última instancia, dotan de direccionalidad y sentido a la sociedad. Asimismo, la mayoría de sus representantes se distancian explícitamente de las perspectivas institucionalistas anteriores, con lo cual han emergido los neologismos nuevo institucionalismo económico (NIE), nuevo institucionalismo político (NIP) y nuevo institucionalismo sociológico (Goodin, 2003; Peters, 2003; Powell \& DiMaggio, 1999), aunque para algunos autores es cuestionable cuán "nuevas" pueden ser estas propuestas (Stichcombe, 1997).

Los desarrollos contemporáneos sobre las instituciones se relacionan estrechamente con el cuestionamiento o rechazo a los modelos del actor racional y la formulación de un modelo de comportamiento humano que acentúa la racionalidad limitada con la cual los hombres actúan en el mundo (Simon, 1989), el alejamiento de las perspectivas hisperholísticas (como el funcional-estructuralismo) de utilidad limitada para dar cuenta de los fenómenos micro y mesociales; y el creciente reconocimiento -en lo cual la teoría de sistemas tuvo una importante influencia- de la creciente complejidad/diferenciación del mundo.

En el ámbito de la sociología, la teoría social mantiene la preocupación por las instituciones y su capacidad para modelar la acción social. Así, son vistas como los engranajes a nivel mesosocial que articulan los procesos macro y microsociales, $y$ tienen como finalidad la internalización, la transmisión y la permanencia de determinadas pautas de representación y acción. En la medida en que los actores sociales son agentes; es decir, son capaces de comprender el mundo, reflexionarlo y transformarlo con su acción (Bourdieu, 2002; Giddens, 1993), el impacto de las instituciones no siempre es unilateral: los actores sociales son capaces de desplegar procesos de resistencia abiertos o larvados, de corta o larga duración; incluso, pueden generar altos grados de oposición en (y en contra de) los marcos institucionales.

La vertiente neoinstitucionalista en sociología también ha tenido un importante desarrollo a partir de los estudios organizacionales (Powell \& DiMaggio, 1999). Los trabajos de mayor influencia en el contexto estadounidense, se debieron a Meyer y Rowan (Meyer, 1977; Meyer \& Rowan, 1977), que dieron lugar a una amplia variedad de análisis sobre los efectos de la cultura, el ritual y la ceremonia en las organizaciones (Powell \& DiMaggio, 1999; Scott, 1998).

Desde esta perspectiva, las instituciones son elementos de las estructuras sociales con cierta autonomía, tienen un profundo carácter histórico y cultural, se articulan generalmente en los niveles intermedios de la sociedad y poseen un anclaje cognitivo en los actores sociales. El NIs postula, además, que los individuos no eligen libremente entre instituciones, sino que las reglas institucionales limitan las opciones, a la par que establecen criterios para definir las preferencias individuales (Powell \& DiMaggio, 1999).

Si nos desplazamos a los análisis institucionales de la economía, el neoinstitucionalismo económico (NIE) se caracteriza por transformar los supuestos de la teoría económica neoclásica estándar para, desde una diversidad de miradas, acentuar la validez heurística del concepto "institución", en el análisis del intercambio económico y político.

$\mathrm{Al}$ tener en cuenta la noción de racionalidad limitada (Simon, 1989), el concepto de costos de transacción (Coase, 1994) y la teoría de la agencia (Alchian \& Demsetz, 1972; Alchian, 1977), puede afirmarse que esta perspectiva ancla la centralidad de las instituciones en ciertas concepciones sobre el comportamiento humano (racionalidad limitada/oportunismo), y en las condiciones en que tienen lugar los intercambios entre los individuos (complejidad/incertidumbre del ambiente, costos de transacción).

Por costos de transacción se comprenden aquellos costos que no emergen directamente del proceso de producción de bienes y servicios: costos de información; negociación; diseño, vigilancia y cumplimiento de contratos; y protección de los derechos de propiedad de las partes en el intercambio. Según el NIE, los costos de transacción siempre son positivos (Ayala, 1996; North, 1990; Williamson, 1991). La relación de agencia implica la aparición del riesgo moral: la posibilidad de que el agente (gestor político o gestor empresarial) busque objetivos 
personales en detrimento de los intereses del principal (el ciudadano o el accionista). Habrá una pérdida de eficiencia siempre que los costos y perjuicios ocasionados por una decisión no recaigan sobre el individuo que decide (Ayala, 1996). Finalmente, el concepto de oportunismo consiste en la procuración con dolo del propio interés; esto es, cuando los individuos realizan promesas falsas o vacías en el intercambio con otros (Williamson, 1991).

En presencia de estos factores, las instituciones nacen y se mantienen en el tiempo para dar certidumbre a las relaciones de intercambio entre los hombres. Estas se pueden comprender, de acuerdo con Douglass North, como "las reglas del juego en una sociedad o, más formalmente, los constreńimientos ideados por los hombres para dar forma a la interacción humana" (North, 1990, p. 3).

Las instituciones reducen la incertidumbre al ofrecer una estructura cotidiana a la interacción, estructuran el sistema de incentivos para el intercambio humano (ya sea económico, político o social), definen y limitan el conjunto de elecciones de los individuos y disminuyen el costo para conocer las violaciones y la severidad del castigo en caso de que los individuos violen las condiciones del intercambio (North, 1990).

Creaciones humanas en el devenir histórico, pueden clasificarse en reglas formales e informales que tienden a perdurar a lo largo de grandes períodos. Dentro de los constreñimientos formales, se encuentran, de manera jerárquica, las constituciones, las leyes y los contratos y tienen como denominador común el hecho de que, por lo general, son escritas.

Las informales son información socialmente transmitida de generación en generación y que forma parte del patrimonio cultural de una sociedad (convenciones, códigos de conducta, normas sociales, etc.) (North, 1990). Adicionalmente, el NIE distingue entre instituciones y organizaciones: las instituciones son las reglas del juego y las organizaciones los jugadores que actúan bajo esas reglas (North, 1990).

Más allá de su eficiencia, las instituciones se sostienen en el tiempo y, por lo general, sus cambios son de carácter incremental; es decir, sin grandes disrupciones. Así, sus características actuales están modeladas, tanto por su trayectoria histórica (path dependence), como por las situaciones, los actores y los intereses que interactúan con ellas en un determinado período histórico (North, 1990).

A través de modelos matemáticos y econométricos estilizados, el NIE ha intentado desarrollar una teoría general de las instituciones -y de la gobernabilidad-, de carácter universal y con gran poder de generalización, susceptible de ser aplicada a múltiples esferas y niveles, así como a diferentes períodos temporales (a corto, mediano y largo plazo).

Sin embargo, subyacen a esta perspectiva varios factores: un sobredimensionamiento del papel de las instituciones para resolver los problemas del intercambio, la asunción de que su aparición y existencia son un suceso aproblemático, su ignorancia del tema del poder, y el no reconocimiento de las limitaciones de las instituciones para dotar de eficiencia a los intercambios en un mundo relativamente ambiguo (Del Castillo, 1997, pp. 15-16).

Williamson subraya la carencia de una teoría unificada de las instituciones, pero señala los aportes hechos con base en una pluralidad de enfoques (Williamson, 2000). También adelanta cuatro dimensiones en el análisis institucional que pueden articular los hallazgos del NIE con los de otras disciplinas sociales: el nivel del enraizamiento social (social embedensess), donde las normas, costumbres y tradiciones se localizan y operan; el nivel de las reglas formales del juego; el nivel de los contratos y transacciones; y el nivel de los mecanismos relacionados con la asignación de recursos (precios y cantidades) (Williamson, 2000, pp. 596-599).

Aunque el análisis institucional en economía se ha enfocado generalmente en los niveles intermedios de análisis (reglas formales y organizaciones, y estructura de gobernación para las transacciones), la propuesta de incluir indagaciones más sistemáticas sobre la influencia de la cultura (reglas informales) en el carácter de la economía en el largo plazo, puede constituirse en una de las propuestas más atractivas y sólidas de los análisis institucionales actuales, con una impronta en la ciencia económica y en la gestación de una propuesta interdisciplinaria que incluya los avances de las diferentes ciencias sociales sobre el tema.

Por último, si no desplazamos a la ciencia política, el término nuevo institucionalismo político (NIP), aunque se debe a March y Olsen (1984), acoge a una miríada de estudios que provienen de diferentes subdisciplinas y enfoques. Por ello, acompańa a esta extraordinaria producción una multiplicidad de miradas y un sinfín de clasificaciones de estos "institucionalismos" (Peters, 2001, 2003; Rohtstein, 2001; Taylor \& Hall, 1996).

Peters (2001) refiere que el NIP se concentra más en las estructuras y en las organizaciones, pero comparte con la revolución conductista la preocupación por la teoría y el desarrollo de métodos analíticos adecuados. También 
se distingue de los institucionalismos anteriores, por la inclusión de las instituciones como variables dependientes e independientes en el análisis.

Además de la idea compartida de que "las instituciones importan", los análisis institucionales en ciencia política toman en cuenta las siguientes consideraciones: 1 . las instituciones son un rasgo estructural de la sociedad; 2. tienen cierta estabilidad en el tiempo; 3. afectan el comportamiento individual; y 4. entre los miembros de las instituciones debe haber cierto sentido de valores compartidos (Peters, 2003).

En ese contexto, una de las propuestas más relevantes es el institucionalismo de la elección racional, que se concentra en el estudio de los microfundamentos del análisis institucional a partir de dos niveles de análisis: el exógeno, que analiza los efectos de las instituciones, y el endógeno, que se centra en explicar por qué las instituciones adoptan ciertas formas y no otras (Weigast, 2001).

Desde esta perspectiva, las instituciones son un sistema de reglas que, al generar información y ofrecer un medio estable a las elecciones y la toma de decisiones, viabilizan la maximización de la utilidad, la agregación de preferencias y, presuntamente, el óptimo social (Knight, 1992). De igual manera, pueden ser consideradas como los límites a los excesos maximizadores de actores sociales como los políticos, los legisladores o los burócratas (Niskanen, 1971).

Las perspectivas de la elección racional así como las corrientes más cercanas al neoinstitucionalismo económico, también se han interesado en la forma como las instituciones conforman los resultados políticos en términos de estabilidad, eficiencia y utilidad social. Estos asuntos han ocupado un lugar central en la ciencia política comparada, que indaga sobre las posibilidades y limitaciones de ciertas instituciones con relación con otras: los regímenes presidencialistas o los parlamentarios, los sistemas políticos federales o los unitarios, los gobiernos divididos, los sistemas electorales y los ciclos de votación, etc. (Cox, 2004; Fiorina, 1992; Lijphart, 1987; Shugart \& Carey, 1992; Weaver \& Rockman, 1993). En su conjunto, dichos estudios han logrado producir evidencias de gran sofisticación y elegancia analítica, que han consolidado la vertiente más "positiva" de la ciencia política.

Adicionalmente, destacan en la disciplina el institucionalismo histórico, que analiza la trayectoria de decisiones anteriores y cómo modelan las instituciones existentes (Krasner, 1984); los estudios institucionales en el área de las relaciones internacionales, preocupados por la conformación de los regímenes internacionales y sus resultados en la cooperación (Keohane, 1989); y el enfoque de políticas públicas, que se concentra en el estudio de los arreglos institucionales, actores y redes en la configuración de las políticas (Peters, 2001).

Por último, debe mencionarse el aporte de March y Olsen en el análisis institucional. Con base en la propuesta de Simon $(1957,1989)$, estos autores definen a las instituciones como un conjunto de reglas y rutinas que establecen las acciones apropiadas en términos de roles y situaciones: cuál situación, qué rol es el adecuado y qué obliga a hacer la situación (March \& Olsen, 1997).

En la medida en que permiten articular las acciones, las instituciones constituyen una estructura de rutinas, funciones y reglas que organizan un proceso político potencialmente desordenado, al conformar el significado y crear un orden interpretativo en el que el comportamiento político puede comprenderse y lograr continuidad. Con ello, cobra centralidad la fuerza ordenadora de los símbolos, los rituales, las ceremonias, los relatos y las dramatizaciones de la vida política, que configuran los comportamientos y las prácticas dentro de esa esfera, lo cual se relaciona más con una lógica de lo adecuado que con la búsqueda de la eficiencia (March \& Olsen, 1997).

\section{A modo de conclusión: logros y nudos problemáticos de los estudios sobre las instituciones}

Este recorrido por los desarrollos más relevantes del concepto "institución", evidencia que, en términos históricos, ha tenido lugar una sofisticación teórica en el tratamiento de las instituciones que, al menos, pone de relieve dos procesos: la importancia indiscutible de los enfoques institucionalistas en el tratamiento de los fenómenos sociales contemporáneos, y la emergencia de articulaciones interdisciplinarias cada vez más necesarias en el estudio de las sociedades del siglo XXI.

En su conjunto, estas perspectivas reconocen que las instituciones son un conjunto de reglas, normas, valores y costumbres que rigen y a la par, son creaciones de la acción social. Tienen la particularidad de durar en el tiempo, aunque pueden aparecer por accidente, evolucionar de manera más o menos "natural" o ser producto de la acción racional -intencionada- de los individuos. En su conjunto, conforman un entramado con distintos niveles y alcances que le dan sentido al orden social y pueden actuar a nivel de la totalidad social o en un área o aspecto específico de la sociedad. 
Ellas son la bisagra entre el nivel macrosocial y el nivel microsocial. Desde ese ámbito, interconectan la cultura con los individuos, lo histórico con lo situacional, lo estructural con lo cognitivo, el orden con la acción. En su accionar, modelan las preferencias, elecciones y el comportamiento de los individuos y les ofrecen recursos para lidiar con la complejidad e incertidumbre del ambiente.

Sin embargo, los análisis institucionales tienen nudos problemáticos que es imprescindible discutir y, eventualmente, superar. En primer lugar, urge un debate profundo sobre qué entender por instituciones, para trascender nociones demasiado abarcadoras o excesivamente reduccionistas como la socorrida frase "reglas del juego", e impulsar conceptos más consistentes y sistemáticos.

En segundo término, el estudio de la interacción entre instituciones y preferencias se ha constituido en un campo problemático, pues existe un punto de ruptura entre las visiones que enfatizan su carácter exógeno y las que postulan que las instituciones configuran las preferencias. Ante ello, se requiere ampliar los estudios empíricos sobre la materia para observar cómo transcurre este proceso, pues algunas investigaciones han constatado que tanto a nivel microsocial como a nivel macrosocial, estructuras institucionales diferentes generan comportamientos diferentes en los individuos (Levi, 1997; Putnam, 1993).

Por último, otro nudo problemático alude al cambio institucional y a la necesidad de construir diseños institucionales que promuevan resultados valiosos socialmente (Goodin, 2003). En ese contexto, una línea de reflexión interesante la provee Elster al apuntar que los diseños institucionales, en vez de basarse en los supuestos racional-instrumentales o en el cálculo de las posibles consecuencias de la decisión, pueden construirse a partir de un discurso deontológico o normativo (Elster, 1991); esto es, siguiendo ciertos criterios morales -como concepciones compartidas sobre la justicia, las normas sobre la igualdad o el tratamiento justo- que viabilicen o al menos permitan reconfigurar las relaciones sociales con base en valores humanos universales.

A pesar de estas limitaciones, los enfoques institucionalistas actualmente existentes, pueden proveer el material necesario para esbozar un proyecto teórico común en el estudio de las instituciones. Si se sigue la propuesta de Williamson (2000), es posible enfrentar este reto organizando el análisis en función de las múltiples dimensiones espaciales y temporales en las que operan las "formas" institucionales. De cierta manera, este ejercicio supone un "viaje a la semilla": significa engarzar los conocimientos fragmentados provenientes de las distintas parcelas disciplinarias en un campo teórico común orientado en el tiempo y el espacio social; un esfuerzo que requiere, en primera instancia, entender al saber social como construcción indivisible.

\section{Referencias}

Alchian, A. A., \& Demsetz, H. (1972). Production, information costs, and economic organization. American Economic Review, 62, 772-775.

Alchian, A. A. (1977). Some economics of property rights. En A. A. Alchian (Eds.), Economic forces at work (pp. 127-149). Indianapolis: Liberty Press.

Arrow, K. (1951). Elección socialy valores individuales. España: Planeta-Agostini.

Ayala, J. (1996). Mercado, elección pública e instituciones. Una revisión de las teorías modernas del estado. México: Porrúa.

Bourdieu, P. (2002). Razones prácticas. Por una teoría de la acción. Madrid: Anagrama.

Brinton, M. C., \& Nee, V. (Eds.). (1998). The New Institutionalism in Sociology. NY: Russell Sage Foundation.

Buchanan, J., \&Tullock, G. (1962). El cálculo del consenso. Fundamentos Lógicos de la democracia constitucional. Barcelona: Planeta-De Agostini.

Caporaso, J., \& Levine, D. (1992). Theories of political economics. United Kingdom: Cambridge University Press.

Coase, R. H. (1994). La empresa, el mercado y la ley. Madrid: Alianza Editorial.

Coleman, J. S. (1988a). Social Capital in the Creation of Human Capital. The American Journal of Sociology, 94, 95-120.

Coleman, J. S. (1988b). The Creation and Destruction of Social Capital: Implications for the Law. Notre Dame Journal of Law, Ethics \& Public Policy, 3, 375-404.

Commons, J. R. (1932). The problems of correlating law, economics and ethics. Wisconsin Law Review, 8, 3-26.

Commons, J. R. (1934). Institutional economics. NY: Macmillan.

Cox, G. (2004). Hacer que los votos cuenten: la coordinación estratégica de los sistemas electorales del mundo. Barcelona: Gedisa.

Del Castillo, A. (1997). El nuevo institucionalismo en el análisis organizacional: conceptos y enunciados explicativos. Documento de trabajo Núm.44, División de Administración Pública. México: CIDE.

Diccionario Jurídico Mexicano (1988). Instituciones. México: Porrúa-UNAM, pp. 1745-1748.

Downs, A. (1957). An economic theory of democracy. NY: Harper and Row. 
Durkheim, E. (2001). Las reglas del método sociológico. México: Fondo de Cultura Económica.

Elster, J. (1991). The possibility of rational politics. En D. Held, (Eds.), Political theory today. (pp. 115-142). Oxford: Polity Press.

Fiorina, M. (1992). Divided government. Manchester: Manchester University Press.

Giddens, A. (1993). Las nuevas reglas del método sociológico: critica positiva de las sociologías interpretativas. Argentina: Amorrortu.

Goodin, R. (2003). Teoría del diseño institucional. Barcelona: Gedisa.

Granovetter, M. (1985). Economic Action and Social Structure: The Problem of Embeddedness. The American Journal of Sociology, 91(3), 481-510

Gruchy, A. G. (1972). Contemporary Economic Thought: The Contribution of Neo-Institutional Economics. NY: Macmillan.

Herrera-Gómez, M., \& Castillo, A. (2004). Generación y transformación de las instituciones sociales; los procesos morfoestáticos y los procesos morfogenéticos. Reis. Revista Española de Investigaciones Sociológicas, 7, 49-87.

Hodgson, G. M. (1998). On the evolution of Thorstein Veblen's evolutionary economics. Cambridge Journal of Economics, $22,415-431$

Homans, G. C. (1961). Social behavior. NY: Harcourt Brace and World.

Keohane, R. O. (Eds.). (1989). International Institutions and State Power: Essays in International Relations Theory. Bouldner, Colorado: Westview Press.

Knight, J. (1992). Institutions and social conflict. Cambridge, UK: Cambridge University Press.

Krasner, S. (1984). Approaches to the State: alternative conceptions and hystorical dynamics. Comparative Politics, 16, 226-246.

Levi, M. (1997), Consent, Dissent, and Patriotism. NY: Cambridge University Press.

Lijphart, A. (1987). Las democracias contemporáneas: un estudio comparativo Barcelona: Ariel.

March, J. G., \& Olsen, J. P. (1984). The New Institucionalism: organizational factors in political life. American Political Science Review, 78, 738-749

March, J. G., \& Olsen, J. P. (1997). El redescubrimiento de las instituciones. La base organizativa de la politica. México: Fondo de Cultura Económica.

Merton, R. K. (1998). Foreword. En M. C. Brinton \& V. Nee, (Eds.), The New Institutionalism in Sociology (pp. 15-19). NY: Russell Sage Foundation.
Meyer, J. W. (1977). The Effects of Education as an Institution. The American Journal of Sociology, 83(1), 55-77.

Meyer, J. W., \& Rowan, B. (1977). Institutionalized Organizations: Formal Structure as Myth and Ceremony. American Journal of Sociology, 83(2), 340-363

Nee, V., \& Ingram, P. (1998). Embeddedness and beyond: Institutions, Exchange, and Social Structure. En M. C. Brinton \& Nee, V. (Eds.), The New Institutionalism in Sociology (pp. 19-45). NY: Russell Sage Foundation.

Niskanen, W. (1971). Bureaucracy and representative government. Chicago: Aldine-Atherton.

North, D. (1990). Institutions, Institutional Change, and Economic Performance. Cambridge: Cambridge University Press.

Olson, M. (1965). The logic of collective action. Public goods and the theory of groups. Cambridge (Mass.): Harvard University Press.

Parsons, T. (1990). Prolegomena to a theory of social institutions. American Sociological Review, 55(3), 319-333.

Parsons, T. (1982), Selected Writings. Chicago: University of Chicago Press.

Peters, B. G. (2001). Las instituciones políticas: lo viejo y lo nuevo. En R. E. Goodin \& H. D. Klingemann (Eds.), Nuevo Manual de Ciencia Política (Tomo I, pp. 304-325). Madrid: Istmo.

Peters, B. G. (2003). El nuevo institucionalismo. Teoría institucional en Ciencia Politica. Barcelona: Gedisa.

Powell, W., \& DiMaggio, P. J. (1999). El nuevo institucionalismo en el análisis institucional. México: Fondo de Cultura Económica.

Putnam, R. (1993). Making Democracy Work: Civic Traditions in Modern Italy. Princenton: Princeton University Press.

Rohtstein, B. (2001). Las instituciones políticas: una visión general. En R. E. Goodin \& Klingemann, H. D. (Eds.), Nuevo Manual de Ciencia Política (Tomo I, pp. 199-246). Madrid: Istmo.

Rutherford, M. (2001). Institutional Economics: then and now. Journal of Economic Perspectives, 15(3), 173-194

Selznick, P. (1957). Leadership and Administration: a sociological interpretation. Illinois: Row, Peterson.

Selznick, P. (1949). TVA and the grass roots. A study in the Sociology of formal organization. Berkeley: University of California Press.

Shepsle, K. A., \& Bonchek, M. S. (2005). Las fórmulas de la política. Instituciones, racionalidad y comportamiento. México: Taurus-CIDE.

Shugart, M. S., \& Carey, J. M. (1992). Presidents and Assemblies. Constitutional Design and Electoral Dynamics. NY: Cambridge University Press. 
Simon, H. (1957). Models of Man, Social and Rational: Mathematical Essays on Rational Human Behavior in a Social Setting. NY: John Wiley and Sons.

Simon, H. (1989). Naturaleza y limites de la razón humana. México: Fondo de Cultura Económica.

Stinchcombe, A. L. (1997). On the Virtues of the Old Institutionalism. Annual Review of Sociology, 23, 1-18.

Vargas, A. (2001). Las Instituciones de Justiniano en Nueva España. México: UNAM.

Veblen, T. (1974). La Clase Ociosa. México: Fondo de Cultura Económica.
Weaver, R. K., \& Rockman, B. A. (1993). Do institutions matter? Washington, DC: Brookings Institution.

Weber, M. (2005). Economía y sociedad. México: Fondo de Cultura Económica.

Weigast, B. (2001). Las instituciones politicas: perspectivas de elección racional. Madrid: Istmo.

Williamson, O. (1991). Mercados y jerarquias: su análisis e implicaciones antitrust. México: Fondo de Cultura Económica.

Williamson, O. (2000). The New Institutional Economics: taking stock, looking ahead. Journal of Economic Literature, 38, 595-613. 